\title{
ON A THEOREM OF N. KATZ AND BASES IN IRREDUCIBLE REPRESENTATIONS
}

\author{
DAVID KAZHDAN \\ Dedicated to the memory of Leon Ehrenpreis
}

1.

Abstract. N. Katz has shown that any irreducible representation of the Galois group of $\mathbb{F}_{q}((t))$ has unique extension to a special representation of the Galois group of $k(t)$ unramified outside 0 and $\infty$ and tamely ramified at $\infty$. In this paper we analyze the number of not necessarily special such extensions and relate this question to a description of bases in irreducible representations of multiplicative groups of division algebras.

Let $k=\mathbb{F}_{q}, q=p^{r}$ be a finite field, $\bar{k}$ the algebraic closure of $k, F:=$ $k((t))$ and $\bar{F}$ be the algebraic closure of $F$. The restriction on $\bar{k} \subset \bar{F}$ defines a group homomorphism

$$
\operatorname{Gal}(\bar{F} / F) \rightarrow \operatorname{Gal}(\bar{k} / k)=\hat{\mathbb{Z}}
$$

and we define the Weil group of the field $F$ as the preimage $\mathcal{G}_{0} \subset$ $\operatorname{Gal}(\bar{F} / F)$ of $\mathbb{Z} \subset \hat{\mathbb{Z}}$ under this homomorphism.

We denote by $\underline{\mathbb{P}}^{1}$ the projective line over $k$, set $E:=k(t)$ and denote by $S$ the set of points of $\underline{\mathbb{P}}^{1}$. For any $s \in S$ we denote by $E_{s}$ the completion of $E$ at $s$. Using the parameter $t$ on $\underline{\mathbb{P}}^{1}$ we identify the fields $E_{0}$ and $E_{\infty}$ with $F$ and therefore identify $\mathcal{G}_{0}$ with the Weil groups of the fields $E_{0}$ and $E_{\infty}$.

Let $\tilde{E}$ be the maximal extension of the field $E$ unramified outside 0 and $\infty$ and tamely ramified at $\infty$. We denote by by $\mathcal{G} \subset \operatorname{Gal}(\tilde{E} / E)$ the Weil group corresponding to the extension $\tilde{E} / E$. We have the natural imbeddings

$$
\mathcal{G}_{0} \hookrightarrow \mathcal{G}, \mathcal{G}_{\infty} \hookrightarrow \mathcal{G}
$$

well defined up to conjugation. Therefore for any complex representation $\rho$ of $\mathcal{G}$ the restrictions to $\mathcal{G}_{0}, \mathcal{G}_{\infty}$ define representations $\rho_{0}, \rho_{\infty}$ of the corresponding local groups. The group $\mathcal{G}$ has a unique maximal 
quotient $\overline{\mathcal{G}}$ such that the Sylow $p$-subgroup of $\overline{\mathcal{G}}$ is normal. As shown by N.Katz $([5])$ the composition $\mathcal{G}_{0} \rightarrow \overline{\mathcal{G}}$ is an isomorphism.

Remark A finite-dimensional irreducible representation $\rho_{0}$ of $\mathcal{G}$ is called special if it factors through a representation of the group $\overline{\mathcal{G}}$. One can restate the theorem of N.Katz by saying that for any irreducible representation $\rho_{0}$ of $\mathcal{G}_{0}$ there exists a unique special representation $\rho_{s p}$ of the group $\mathcal{G}$ whose restriction to $\mathcal{G}_{0}$ is equivariant to $\rho_{0}$.

Let $D_{0}$ be a skew-field with center $F, \operatorname{dim}_{F} D_{0}=n^{2}, G_{0}:=D_{0}^{*}$ be the multiplicative group of $D_{F}$ and $\rho_{0}$ be an $n$-dimensional indecomposable representation of the group $\mathcal{G}_{0}$.

Definition 1.1. a) We denote by $\tilde{\sigma}\left(\rho_{0}\right)$ the irreducible discrete series representation of the group $G L_{n}(F)$ which corresponds to $\rho_{0}$ under the local Langlands correspondence ( see for example ([3] ) and by $\sigma\left(\rho_{0}\right)$ the irreducible representation of the group $G_{0}$ which corresponds to $\tilde{\sigma}\left(\rho_{0}\right)$ as in [1].

b) We denote by $r\left(\rho_{0}\right)$ the formal dimension of the representation $\tilde{\sigma}\left(\rho_{0}\right)$ where the formal dimension is normalized in such a way that the formal dimension of the Steinberg representation is equal to 1 . Analogously for any indecomposable representation $\rho_{\infty}$ of the group $\mathcal{G}_{\infty}$ we define an integer $r\left(\rho_{\infty}\right)$.

c) We denote by $A\left(\rho_{0}\right)$ the set of equivalence classes of $n$-dimensional irreducible representations $\rho$ of the group $\mathcal{G}$ whose restriction to $\mathcal{G}_{0}$ is equivalent to $\rho_{0}$ and the restriction to $\mathcal{G}_{\infty}$ is indecomposable.

Theorem 1.2. For any $n$-dimensional irreducible $\overline{\mathbb{Q}}_{l}$-representation of the group $\mathcal{G}_{0}$ the sum $\sum_{\rho \in A\left(\rho_{0}\right)} r\left(\rho_{\infty}\right)$ is equal to $r\left(\rho_{0}\right)$.

Proof. Let $\mathbb{A}=\prod_{s \in S} E_{s}$ the ring of adeles of $E$ and $D$ be a skewfield with center $E$ unramified outside $\{0, \infty\}, D_{0}:=D \otimes_{E} E_{0}$ and $D_{\infty}:=D \otimes_{E} E_{\infty}$. Then $D_{0}, D_{\infty}$ are local skew-fields. Let $\underline{G}$ be the multiplicative group of $D$ considered as an the algebraic $E$-group.

It follows from [6] that we can identify the set $A\left(\rho_{0}\right)$ with the set of automorphic representations $\tilde{\pi}=\prod_{s \in S} \tilde{\pi}_{s}$ of the group $G L_{n}(\mathbb{A})$ such that the representation $\tilde{\pi_{0}}$ is equivalent to $\tilde{\sigma}\left(\rho_{0}\right)$ and the representation $\tilde{\pi}_{\infty}$ is of discrete series. Then it follows from [1] that we can identify the set $A\left(\rho_{0}\right)$ with the set of automorphic representations $\pi=\prod_{s \in S} \pi_{s}$ of the group $\underline{G}(\mathbb{A})$ such that the representation $\pi_{0}$ is equivalent to $\sigma\left(\rho_{0}\right)$. We will use this identification for the proof of the Theorem 1.2. 
ON A THEOREM OF N. KATZ AND BASES IN IRREDUCIBLE REPRESENTATIONS3

We denote by $N: D_{0} \rightarrow F$ the reduced norm and define

$$
\mu:=\nu \circ N: D_{0}^{*} \rightarrow \mathbb{Z}, K_{0}:=\mu^{-1}(0)
$$

where $\nu: F^{*} \rightarrow \mathbb{Z}$ is the valuation. Then $K_{0} \subset D_{0}^{*}$ is a maximal compact subgroup. We define the first congruence subgroup $K_{0}^{1}$ by

$$
K_{0}^{1}:=\left\{k \in K_{0} \mid \mu(k-I d)>0\right\}
$$

As is well known $K_{0}^{1}$ is a normal subgroup of $D_{0}^{*}$ such that $K_{0} / K_{0}^{1}=\mathbb{F}_{q^{n}}^{*}$ and $D_{0}^{*} / K_{0}^{1}=\mathbb{Z} \ltimes \mathbb{F}_{q^{n}}^{*}$ where $\mathbb{Z}$ acts on $\mathbb{F}_{q^{n}}^{*}$ by $(n, x) \rightarrow x^{q^{n}}$.

For any $s \in S-\{0, \infty\}$ we identify the group $G_{E_{s}}$ with $G L\left(n, E_{s}\right)$ and define $K_{s}:=G L\left(n, \mathcal{O}_{s}\right)$. We write $G_{\mathbb{A}}:=D_{\infty}^{*} \times G L_{n}\left(\mathbb{A}^{0}\right)$ where

$$
G L_{n}\left(\mathbb{A}^{\infty}\right):=D_{0}^{*} \times \prod_{s \in S-\{0, \infty\}} G L\left(n, E_{s}\right)
$$

and define

$$
K^{0}:=\prod_{s \in S-\{0, \infty\}} K_{s} \times K_{E_{\infty}}, K^{1}:=\prod_{s \in S-\{0, \infty\}} K_{s} \times K_{E_{\infty}}^{1}
$$

where $K_{E_{\infty}}^{1} \subset K_{E_{\infty}} \subset D_{\infty}^{*}$ is the first congruence subgroup of $G_{\infty}$.

For any irreducible representation $\pi$ of the group $G_{0}$ we denote by $\tilde{\pi}$ the discrete series representation of the group $G L_{n}(F)$ corresponding to $\tilde{\pi}$ as in [1].

Lemma 1.3. a) For any irreducible complex representation $\kappa: D_{0}^{*} / K_{0}^{1} \rightarrow$ Aut $(W)$ and any character $\chi: K_{0} / K_{0}^{1} \rightarrow \mathbb{C}^{*}$ we have

$$
\operatorname{dim}\left(W^{\chi}\right) \leq 1
$$

where $W^{\chi}=\left\{w \in W \mid \kappa(k) w=\chi(k) w, k \in K_{0}\right\}$.

b) For any irreducible representation $\pi$ of the group $G_{0}$ the formal dimension of $\tilde{\pi}$ is equal to the dimension of $\pi$.

Proof. Part a) follows from the isomorphism $D_{0}^{*} / K_{0}^{1}=\mathbb{Z} \ltimes \mathbb{F}_{q^{*}}$.

Part b) follows from [1].

We see that the following equality implies the validity of the Theorem 1.2 .

Claim 1.4. For any $n$-dimensional irreducible $\overline{\mathbb{Q}}_{l}$-representation of the group $\mathcal{G}_{0}$ the sum $\sum_{\pi \in A\left(\rho_{0}\right)} \operatorname{dim}\left(\pi_{\infty}\right)$ is equal to $\operatorname{dim}\left(\sigma\left(\rho_{0}\right)\right)$.

The proof of Claim is based on the following result.

Proposition 1.5. The product map $D_{0}^{*} \times K^{1} \times G_{E} \rightarrow G_{\mathbb{A}}$ is a bijection. 
Proof of the Proposition. The surjectivity follows from Lemma 7.4 in [4]. To show the injectivity it is sufficient to check the equality

$$
\left(D_{0}^{*} \times K^{1}\right) \cap G_{E}=\{e\}
$$

which is obvious

We denote by $\mathbb{C}\left(G_{\mathbb{A}} / G_{E}\right)$ the space of locally constant functions on $G_{\mathbb{A}} / G_{E}$ with compact support, by $\mathbb{C}\left(G_{0}\right)$ the space of locally constant functions on $G_{0}$ with compact support and by $L \subset \mathbb{C}\left(G_{\mathbb{A}} / G_{E}\right)$ the subspace of $K^{1}$-invariant functions. The group $G_{0} \times D_{\infty}^{\star} / K_{\infty}^{1}$ acts naturally on $L$.

Let $\rho_{0}$ be an indecomposable representation of the group $\mathcal{G}_{0}$. We denote by $\left(\sigma\left(\rho_{0}\right), V\left(\rho_{0}\right)\right)$ the corresponding representation of the group $G_{0}$ and identify the set $A\left(\rho_{0}\right)$ with the set of automorphic representations $\pi^{a}=\prod_{s \in S} \pi_{s}^{a}$ of the group $\underline{G}(\mathbb{A})$ such that the representation $\pi_{0}^{a}$ is equivalent to $\sigma\left(\rho_{0}\right)$ and the representation $\pi_{\infty}^{a}$ is trivial on $K_{\infty}^{1}$. Let

$$
\mathcal{H}:=\prod_{s \in S-\{0, \infty\}} \mathcal{H}_{s}
$$

where $\mathcal{H}_{s}$ is the spherical Hecke algebra for $G\left(F_{s}\right)=G L\left(n, F_{s}\right)$. By construction, the commutative algebra $\mathcal{H}$ acts on the $D_{0}^{\star} \times D_{\infty}^{\star} / K^{1}$ module $L$. For any $a \in A\left(\rho_{0}\right)$ we define

$L_{a}:=\operatorname{Hom}_{G_{\mathbb{A}}^{\infty}}\left(\pi^{a}, \mathbb{C}\left(G_{\mathbb{A}} / G_{E}\right)\right)=\operatorname{Hom}_{G_{0} \times \mathcal{H}}\left(\sigma\left(\rho_{0}\right), L\right) \subset \operatorname{Hom}_{G_{0}}\left(\sigma\left(\rho_{0}\right), L\right)$

Lemma 1.6. a) The restriction $r: L \rightarrow \mathbb{C}\left(G_{0}\right)$ is an isomorphism of $G_{0}$-modules where $G_{0}$ acts on $\mathbb{C}\left(D_{0}^{*}\right)$ by left translation.

b) $\operatorname{Hom}_{G_{0}}\left(\sigma\left(\rho_{0}\right), L\right)=V^{\vee}$ where $V^{\vee}$ is the dual space to $V\left(\rho_{0}\right)$.

c) $V^{\vee}=\oplus L_{a}, a \in A\left(\rho_{0}\right)$ where the algebra $\mathcal{H}$ acts on $L_{a}, a \in A\left(\rho_{0}\right)$ by a character $\chi_{a}: \mathcal{H} \rightarrow \overline{\mathbb{Q}}_{l}^{\star}, \chi_{a} \neq \chi_{a^{\prime}}$ for $a \neq a^{\prime}$ and the representations $\pi_{\infty}^{a}$ of the group $D_{\infty}^{\star} / K^{1}$ on $M_{a}$ are irreducible.

d) The representations $\pi_{\infty}^{a}$ are associated with the restriction $\rho(a)_{\infty}$ by the local Langlands correspondence.

Proof. The Lemma follows immediately from the Proposition and the strong multiplicity one theorem ([7]and [1])

This Lemma implies the validity of Claim and therefore of Theorem 1.2. Indeed we have

$$
\operatorname{dim}(V)=\operatorname{dim}\left(V^{\vee}\right)=\sum_{a \in A\left(\rho_{0}\right)} \operatorname{dim}\left(L_{a}\right)=\sum_{a \in A\left(\rho_{0}\right)} \operatorname{dim}\left(\pi_{\infty}^{a}\right)=\sum_{a \in A\left(\rho_{0}\right)} r\left(\rho(a)_{\infty}\right) \square
$$


One can ask whether one can extend Theorem 1.2 to the case of other groups. More precisely, let $G$ be a split reductive group with a connected center and ${ }^{L} G$ be the Langlands dual group. Consider a homomorphism $\rho_{0}: \mathcal{G}_{0} \rightarrow{ }^{L} G$ such that the connected component of the centralizer $Z_{\rho}:=Z_{L_{G}}(\operatorname{Im}(\rho))$ is unipotent. Let $\left[Z_{\rho}\right]$ be the group of connected components of the centralizer $Z_{\rho}$. Conjecturally, one can associate with $\rho_{0}$ an $L$-packet of irreducible representations $\pi_{\rho}(\tau)$ of the group $G_{0}:=G(F)$ parameterized by irreducible representations $\tau$ of $\left[Z_{\rho}\right]$ and there exists an integer $r\left(\rho_{0}\right)$ such that the formal dimension of $\pi_{\rho}(\tau)$ is equal to $r(\rho) \operatorname{dim}(\tau)$.

We denote by $A^{G}\left(\rho_{0}\right)$ the set of conjugacy classes of homomorphisms $\rho: \mathcal{G} \rightarrow{ }^{L} G$ whose restriction on $\mathcal{G}_{0}$ is conjugate to $\rho_{0}$ and such that the connected component of the centralizer of the restriction on $\mathcal{G}_{\infty}$ is unipotent.

Question. Is it true that $r\left(\rho_{0}\right)=\sum_{a \in A\left(\rho_{0}\right)} r\left(\rho_{\infty}\right)$ where $r\left(\rho_{\infty}\right)$ is defined in the same way as $r\left(\rho_{0}\right)$ ?

2.

Let $G$ be a reductive group over a local field. As is well known one can realize the spherical Hecke algebra $\mathcal{H}$ of $G$ geometrically, that is as the Grothendick group of the monoidal category of perverse sheaves on the affine Grassmanian. Analogously in the case when $G$ be a reductive group over a global field of positive characteristic the unramified geometric Langlands conjecture predicts the existence of a geometric realization of the corresponding space of automorphic functions.

Let $\underline{C}$ be a smooth absolutely irreducible $\mathbb{F}_{q}$-curve, $q=p^{m}, S$ be the set of geometric points of $\underline{C}, \Gamma:=\pi_{1}(\underline{C})$. For any $s \in S$ we denote by $F r_{s} \subset \Gamma$ the conjugacy class of the Frobenius at $s$.

Let $E$ be the field of rational functions on $\underline{C}$. For any $s \in S$ we denote by $E_{s}$ the completion of $E$ at $s$ and we denote by $\mathbb{A}$ be the ring of adeles of $E$. Fix a prime number $l \neq p$.

Let $\underline{G}$ be a split reductive group, and $\hat{K}:=\prod_{s \in S} G\left(\mathcal{O}_{s}\right) \subset G(\mathbb{A})$ be the standard maximal compact subgroup. An irreducible representation $(\pi, V)=\otimes_{s \in S}\left(\pi_{s}, V_{s}\right)$ of $G(\mathbb{A})$ is unramified if $V^{\hat{K}} \neq\{0\}$. In this case $\operatorname{dim}\left(V^{\hat{K}}\right)=1$. So for any unramified representation $(\pi, V)$ of the group $G(\mathbb{A})$ there is a special spherical vector $v_{s p} \in V$ defined up to a multiplication by a scalar. 
Let ${ }^{L} G$ be the Langlands dual group and $\rho$ a homomorphism from $\Gamma$ to ${ }^{L} G\left(\overline{\mathbb{Q}}_{l}\right)$ such that for any $s \in S$ the conjugacy class $\gamma_{s}:=\rho\left(F r_{s}\right) \subset$ ${ }^{L} G\left(\overline{\mathbb{Q}}_{l}\right)$ is semisimple. In such a case we can define unramified representations $\left(\pi_{\gamma_{s}}, V_{s}\right)$ of local groups $G\left(E_{s}\right)$ and the representation $\left(\pi(\rho), V_{\rho}\right)=\otimes_{s}\left(\pi_{\gamma_{s}}, V_{s}\right)$ of the adelic group $G(\mathbb{A})$. According to the unramified geometric Langlands conjecture the homomorphism $\rho$ defines [at least in the case when $\rho$ is tempered] an imbedding

$$
i_{\rho}: V_{\rho} \rightarrow \overline{\mathbb{Q}}_{l}(K \backslash G(\mathbb{A}) / G(E))
$$

and a function $f_{\rho}:=i_{\rho}\left(v_{s p}\right)$ which is defined up to a multiplication by a scalar.

We can identify the set $K \backslash G(\mathbb{A}) / G(E)$ with the set of $\mathbb{F}_{q}$-points of the stack $\mathcal{B}_{G}$ of principal $G$-bundles on $\underline{C}$ and the unramified geometric Langlands correspondence predicts the existence of a perverse Weil sheaf $\mathcal{F}(\rho)$ on $\mathcal{B}_{G}$ such that the function $f_{\rho}$ is given by the trace of the Frobenius automorphisms on stalks of $\mathcal{F}(\rho)$. ( See [2])

If one considers ramified automorphic representations $(\pi, V)=\otimes_{s \in S}\left(\pi_{s}, V_{s}\right)$ of $G(\mathbb{A})$ then there is no natural way to choose a special vector in $V$. So on the "geometric" side one expects not an object $\mathcal{F}(\rho)$ but an abelian category $\mathcal{C}(\rho)$ which is a product of local categories $\mathcal{C}\left(\rho_{s}\right)$ such that the Grothendick K-group of the category $\left[\mathcal{C}\left(\rho_{s}\right)\right]$ coincides with the subspace $V_{s}^{0}$ of the minimal $K$-type vectors of the space $V_{s}$ of the local representation. Such geometric realization of the space $V_{s}^{0}$ would define a special basis of vector spaces $V_{s}^{0}$ which would be a non-archimedian analog of Lusztig's canonical basis. Here we consider only the case of an anisotropic group when the minimal $K$-type subspace $V_{s}^{0}$ coincides with the space $V_{s}$ of the representation of $G$. Moreover we will only discuss a slightly weaker data of a projective basis where a projective basis in a finite-dimensional vector space $T$ is a decomposition of the space $T$ in a direct sum of one-dimensional subspaces. So one could look for a special basis of vector spaces $V_{s}$ which would be a non-archimedian analog of the Lusztig's canonical basis.

Let as before $F:=k((t)), D_{0}$ be a skew-field with center $F, \operatorname{dim}_{0} D_{0}=$ $n^{2}, G_{0}$ be the multiplicative group of $D_{0}$ and $\sigma: G_{0} \rightarrow A u t(V)$ a complex irreducible continuous representation of the group $G_{0}$.

Theorem 2.1. For any irreducible representation $\tau: D_{F}^{*} \rightarrow$ Aut $(T)$ of the group $D_{F}^{*}$ there exists a "natural" projective basis $=\oplus_{a} T_{a}$ of $T$.

Remark 2.2. The construction is global. In particular I don't know how to define a projective basis in the case when $F$ is a local field of 
characteristic zero. It would be very interesting to find a local construction of a projective basis.

The construction. As follows from Lemma $1.6 \mathrm{c})$ we have a decomposition $V^{\vee}=\sum_{a \in A\left(\rho_{0}\right)} M_{a}$ where the group $D_{\infty}^{\star} / K_{\infty}^{1}$ acts irreducibly on $M_{a}$. Therefore the group $\mathbb{F}_{q^{n}}^{\star}=K_{\infty} / K_{\infty}^{1}$ acts on $M_{a}$ and we have a decomposition of $M_{a}$ into the sum of eigenspaces for the action of the group $\mathbb{F}_{q^{n}}^{\star}$. As follows from Lemma 3 a) these eigenspaces are one-dimensional.

\section{REFERENCES}

[1] Deligne, P.; Kazhdan, D.; Vignéras, M.-F. Représentations des alge'bres centrales simples $p$-adiques. Representations of reductive groups over a local field, 33-117, Travaux en Cours, Hermann, Paris, 1984"

[2] Gaitsgory, D. Informal introduction to geometric Langlands. An introduction to Langlands program. 269-281 Burhauser Boston, Boston MA 2003

[3] Henniart, G., Une preuve simple des conjectures de Langlands pour GL(n) sur un corps p-adique, Invent. Math., (2000).

[4] Hrushovski, E,; Kazhdan D.; Motivis Poisson summation. Moscow Math. J. 9(2009) no. 3 569-623

[5] Katz, N.Local-to-global extensions of representations of fundamental groups. (French summary) Ann. Inst. Fourier (Grenoble) 36 (1986), no. 4, 69106.

[6] Lafforgue, L. Chtoucas de Drinfeld et correspondance de Langlands. Invent. Math. 147 (2002), no. 1, 1241.

[7] Piatetskii-Shapiro I. Multiplicity one theorems, Proc. Sympos. Pure Math., vol. 33, Part I, Providence, R. I., 1979, pp. 209-212.

Institute of Mathematics, The Hebrew University, Jerusalem, IsRAEL

E-mail address: kazhdan@math.huji.ac.il 